\title{
¿CÓMO POTENCIAR LA INNOVACIÓN EN CHILE?*
}

\author{
Felipe Larraín B.
}

El crecimiento económico de los países depende crucialmente de cómo evolucione la productividad total de los factores productivos (PTF). A su vez, la expansión de la PTF está estrechamente relacionada a la inversión en investigación y desarrollo (I+D). En este sentido, la economía chilena exhibe hoy niveles de I+D bastante exiguos, que no se han podido superar a pesar de haber estado en la discusión pública y en las prioridades gubernamentales por largos años. Actualmente la inversión en I+D en Chile corresponde a entre 0,6\% y 0,7\% del PIB. En cambio, países exitosos como Finlandia y Corea tienen niveles de I+D que superan el 3\% del PIB.

En este artículo se estudia la importancia de la inversión en investigación y desarrollo para el crecimiento y desarrollo futuro de la economía chilena. Para ello se realiza un análisis de la evidencia empírica y se compara la situación actual del gasto en I+D con la experiencia internacional. El trabajo se enfoca en factores tales como el nivel de I+D, sus fuentes de financiamiento (público o privado) y la composición de este gasto entre investigación básica y aplicada. Por último, se plantean una serie de propuestas dirigidas a incentivar la inversión en I+D.

Felipe Larraín Bascuñán. Profesor Titular, Instituto de Economía, Pontificia Universidad Católica de Chile.

* Este trabajo contó con el financiamiento del Banco Interamericano de Desarrollo (BID). Agradezco la muy eficiente colaboración de Francisco Parro y Felipe Varas y los comentarios de Raúl Rivera y un árbitro anónimo de Estudios Públicos. Como es usual, el contenido de este documento es de exclusiva responsabilidad del autor y no representa necesariamente al BID.

Estudios Públicos, 104 (primavera 2006). 


\section{Introducción}

$\mathrm{L}$

literatura empírica sobre crecimiento económico es enfática en señalar que más de la mitad de las diferencias del nivel y crecimiento del ingreso per cápita entre países se explican por diferencias en la productividad total de factores (PTF). A su vez, el cambio tecnológico es la principal fuente del crecimiento de la productividad en el largo plazo. La forma más utilizada para medir el esfuerzo que un país hace en materia de innovación es el gasto en I+D que realiza. Esta variable mide el esfuerzo sistemático de un país para crear nuevos productos o procesos y para adoptar y adaptar tecnologías, lo que es clave para promover incrementos de productividad. A su vez, estos últimos pueden deberse a mejoras producto de un acercamiento a la frontera tecnológica o de un movimiento de la frontera.

Los niveles actuales de actividad en investigación y desarrollo son muy disímiles entre las distintas economías del mundo. Países líderes en innovación como Finlandia y Corea presentan niveles de gasto que llegan a superar el 3\% del PIB; en otras economías, como Argentina y Grecia, dicha cifra es apenas alrededor de 0,5\% del PIB; en Chile corresponde a una cifra entre 0,6 y $0,7 \%$ del PIB. Dichos niveles de gasto parecen bajos considerando la alta rentabilidad social de este tipo de actividades. La apertura comercial y la inversión externa de las economías menos desarrolladas aparecen como factores claves no sólo para incentivar un mayor esfuerzo en I+D, sino también para facilitar los derrames de conocimientos desde los países que realizan la mayor parte del gasto en I+D a nivel mundial.

Adicionalmente, la evidencia internacional muestra que los países exitosos en innovación parecen tener un sesgo hacia la investigación aplicada, financiada mayormente por el sector privado, lo cual permite una mayor adecuación entre innovación y las necesidades de las empresas.

El diagnóstico para Chile muestra un bajo nivel agregado de gasto en I+D en comparación con los países desarrollados y una baja participación del sector privado en el financiamiento y ejecución de la I+D. Además, el Sistema Nacional de Innovación chileno evidencia fallas de coordinación y poca integración, lo cual resta eficiencia al ya escaso esfuerzo realizado en actividades de innovación.

El objetivo de este trabajo es analizar la actividad de innovación, proponer alternativas de políticas que permitan incrementar el nivel de investigación y desarrollo de Chile y promover una mayor integración del Sistema Nacional de Innovación. Además, se analizan los tipos de incentivos que permiten profundizar la participación del sector privado. 
El trabajo se organiza como sigue. La sección 2 presenta evidencia empírica y teórica de la importancia de la I+D para fomentar el crecimiento económico. Asimismo, analiza los canales a través de los cuales la apertura afecta el gasto en I+D. La sección 3 realiza una comparación internacional del nivel agregado de $\mathrm{I}+\mathrm{D}$, la forma en que se financia, el tipo de investigación y la rentabilidad social y privada de este tipo de actividades. Por su parte, la sección 4 presenta un diagnóstico del esfuerzo actual de Chile en I+D, describe brevemente el Sistema Nacional de Innovación chileno y realiza una propuesta de fomento a la innovación en Chile. Finalmente, la sección 5 presenta las principales conclusiones del trabajo.

\section{Inversión en I+D y crecimiento}

\subsection{Crecimiento y productividad}

Hoy en día existe una gran dispersión en los estándares de vida de las naciones. Países como Estados Unidos tienen un ingreso per cápita anual de casi US\$ 40.000, mientras que otros como Burundi apenas llegan a los \$90. La literatura empírica sobre crecimiento económico muestra que más de la mitad de las diferencias de ingreso per cápita y crecimiento entre las naciones se explican por diferencias en la productividad total de factores $(\mathrm{PTF})^{1}$. A su vez, el cambio tecnológico muestra ser la principal fuente del crecimiento de la productividad en el largo plazo. Esto porque a diferencia del aumento en el stock de capital o trabajo, el progreso técnico no exhibe rendimientos decrecientes ${ }^{2}$.

Por su parte, existe un amplio consenso sobre la importancia que tiene la inversión en $\mathrm{I}+\mathrm{D}$ en el crecimiento de la productividad total de factores y, a través de ello, en la expansión del producto de un país. Países que dedican un mayor porcentaje del PIB a I+D tienden a crecer más rápidamente $^{3}$. La I+D permite a los países adoptar mejores tecnologías, proporciona nuevos y mejores bienes y los frutos de esta actividad se difunden al resto de la economía. La evidencia empírica y los desarrollos teóricos respaldan esta idea.

Levine (2002).

${ }^{1}$ Véase, por ejemplo, Hall y Jones (1999), Dollar y Wolf (1997), Easterly y

${ }^{2}$ Véase Romer (1986).

${ }^{3}$ Al respecto, véase Lichtenberg y Siegel (1991), Bayoumi, Coe y Helpman (1999), Cameron, Proudman y Redding (2005). 


\subsection{Productividad e I+D: Literatura teórica}

Basado en un modelo teórico de crecimiento endógeno, Romer (1990) muestra que la inversión en I+D puede generar importantes externalidades que hacen que los frutos de esta actividad se difundan al resto de la economía. El progreso de la tecnología disponible tiene lugar tanto en el caso de la producción de nuevos bienes como en la mejora de los procesos de producción y en la diferenciación de productos; ello produce un aumento de las tasas de productividad de las empresas que la utilizan y de la economía en su conjunto. La inversión en I+D permite mejorar los resultados de la empresa que la realiza por disminución de costes unitarios de producción, mejora de la calidad de sus productos, o introducción de nuevas variedades de productos, lo que incentiva a innovar. Sin embargo, dadas las dificultades para mantener los derechos de propiedad intelectual, esas mejoras acaban beneficiando a otras empresas y a la economía en su conjunto, a través del incremento del acervo público de conocimiento técnico. Esta externalidad positiva que no captura el innovador explica por qué se sub-invierte en $\mathrm{I}+\mathrm{D}$ y justifica algún tipo de incentivo público a esta actividad.

Por su parte, Grossman y Helpman (1991a, 1991b) y Aghion y Howitt (1992) desarrollan modelos alternativos en donde cada producto nuevo es altamente sustituible por un producto similar de menor calidad. Sobre esta base, la calidad existente de productos produce un benchmark a partir del cual los innovadores intentarán mayores mejoras en la calidad del producto, lo cual produce un spillover de conocimiento desde actuales hacia futuros innovadores. La probabilidad de éxito dependerá de los recursos destinados a I+D. A su vez, la búsqueda de ganancias incentiva la investigación y los innovadores se benefician de observar los éxitos de sus rivales. El proceso resultante es uno de “destrucción-creativa” schumpeteriana, donde productos de alta calidad destruyen las oportunidades de mercado para los productos viejos de menor calidad. Esto, a su vez, induce a un crecimiento de la productividad en el tiempo producto de las mejoras de calidad.

Así, las teorías de crecimiento enfatizan el importante rol de la inversión en I+D sobre el crecimiento económico de un país. Dicho efecto, se produce través de dos canales: primero, permite producir nuevos y mejores bienes, y segundo, permite incrementar el stock de conocimiento disponible para investigación y desarrollo, permitiendo a los países adoptar mejores tecnologías. 


\subsection{Productividad e I+D: Evidencia empírica}

A nivel empírico, son diversos los estudios que encuentran efectos significativo del gasto en I+D sobre la productividad. En un trabajo reciente, Griffith, Redding y Van Reenan (2004) encuentran que la inversión en investigación y desarrollo es fundamental para explicar la convergencia de la tasa de crecimiento de la PTF entre los distintos países de la OECD. Estos autores reportan que el efecto de un aumento de $1 \%$ en la I+D incrementa el crecimiento de la productividad entre $0,4 \%$ y $0,9 \%$.

Para enfatizar la relevancia de aumentar la eficiencia en innovación, Bergoeing y Repetto (2004) analizan las causas que explican la diferencia sustancial de ingresos per cápita entre Chile y Estados Unidos. Concluyen que no es el nivel de inversión por trabajador lo que explica que Chile tenga un menor ingreso por habitante que Estados Unidos. De hecho, sólo un 9\% de esta diferencia se explica por menor inversión. Si Chile y Estados Unidos tuvieran el mismo stock de capital por trabajador, nuestro ingreso per cápita sería todavía un quinto del de Estados Unidos. La diferencia se explica, principalmente, por la menor eficiencia en el uso de tecnologías en Chile. Si con el nivel actual de capital por trabajador Chile utilizara las tecnologías de la misma manera a como lo hace Estados Unidos, su producto per cápita superaría los 25 mil dólares.

\subsection{I+D y apertura}

La relación entre I+D y apertura tiene dos aristas. Primero una mayor apertura comercial puede afectar el esfuerzo en $\mathrm{I}+\mathrm{D}$ realizado por un país. Segundo, el esfuerzo en I+D de un grupo de países puede afectar la productividad de otras economías, convirtiéndose en una fuerza de convergencia en los estándares de vida de los países.

La literatura moderna de comercio internacional ha identificado un número de canales a través de los cuales la apertura comercial afecta el nivel de gasto de I+D de los países. Grossman y Helpman (1991b) identifican cuatro canales. Primero, mayor apertura comercial permite acceder a un mercado más amplio lo cual incentiva un mayor gasto en I+D. Segundo, mayor apertura comercial expone a las empresas domésticas a la competencia extranjera. Aghion, Harris, Howitt y Vickers (2001) demuestran que la exposición a una mayor competencia con las firmas externas afectaría de dos maneras los incentivos a innovar. Por una parte reduce las ganancias de las firmas domésticas y con ello los incentivos para innovar. Al mismo tiempo, una mayor competencia incentiva a las firmas a distanciarse de los "rivales" 
más cercanos, lo cual pueden lograrlo con un mayor esfuerzo en I+D. Los autores concluyen que el segundo canal tendría un impacto mayor, por lo que existiría una relación positiva entre mayor competencia y la tasa de innovación de una economía.

Tercero, mayor apertura comercial elimina redundancia en I+D. Cuando una firma opera en una economía cerrada se encuentra aislada de las empresas extranjeras. Por ende, una firma operando en una economía cerrada intentará desarrollar productos que no son elaborados por otras firmas de su propio país. Dichas empresas no tendrán incentivos para diferenciar su producto de bienes que son producidos en otros países, ya que no esperan competir con productores extranjeros en el mercado doméstico. Como resultado de esto puede haber una duplicación del esfuerzo en I+D. Cuando los países se abren al comercio internacional, cada firma compite con todos los productores del mundo. Bajo dichas circunstancias, una empresa tiene incentivos para diferenciarse de todos los otros productos, externos e internos. Esto minimiza la duplicación de esfuerzos en I+D y con ello incrementa el crecimiento del stock de conocimientos al disminuir los costos de realizar investigación, pues el punto de partida para la I+D será un stock de conocimientos mayor.

Cuarto, y relacionado con lo anterior, la apertura comercial permite compartir entre los países el stock de conocimientos. Esto es, la I+D de un país aumenta el stock de conocimientos disponible para actividades de innovación de ese y otros países en presencia de economías abiertas. Esto, en definitiva, disminuye el costo del esfuerzo en I+D y con ello incentiva un mayor gasto en estas actividades.

Tan importante como entender por qué la apertura comercial afecta el esfuerzo en I+D que efectúan los países, es analizar cómo los flujos de comercio permiten que el esfuerzo en I+D que realizan algunos países afecte la productividad de otros países. La importancia de esto es que más del 95\% del gasto mundial en I+D es ejecutado por las principales economías industriales. De no existir estos spillovers de conocimiento en economías abiertas, el conocimiento generado por las actividades de I+D se difundirían sólo en las economías que llevan a cabo dicho gasto y, por ende, se convertirían en una importante fuente de divergencia en los estándares de vida de los países. Por el contrario, si la apertura comercial permite que el conocimiento se derrame al resto de los países, ésta se convertiría en una fuerza de convergencia entre las economías del mundo.

Coe y Helpman (1995) estimaron los efectos del stock de capital de I+D doméstico y extranjero sobre el nivel de productividad de un país. Los autores concluyen que las economías más abiertas presentan mayores nive- 
les de productividad y que la presencia de I+D extranjera influencia positivamente las ganancias de productividad de dichas economías.

Coe, Helpman y Hoffmaister (1997) estiman el impacto del stock de I+D extranjero de una economía sobre la productividad total de factores en ella para una muestra de 77 países en desarrollo. Esto es, analizan si los países en desarrollo se benefician del esfuerzo de I+D ejecutado por los países industriales. Su principal conclusión es que el stock de I+D de un socio comercial tiene un impacto positivo y significativo sobre la PTF del país local.

Por su parte, Keller (2001) descompuso los spillovers internacionales de I+D en tres partes: comercio, inversión extranjera directa y habilidades de lenguaje. Concluye que cerca del 70\% de los efectos se deben al comercio, $15 \%$ a la inversión extranjera directa y el 15\% restante a las habilidades de lenguaje.

Así, la literatura empírica enfatiza la importancia de la apertura comercial; primero, en incentivar un mayor esfuerzo en I+D en los países y segundo, en facilitar el flujo de conocimientos entre las economías con mayor stock de I+D hacia el resto de los países, afectando así positivamente la productividad de dichos países.

\section{Inversión en I+D: Una comparación internacional}

\subsection{Nivel agregado de I+D y desarrollo}

El Cuadro $\mathrm{N}^{\mathrm{o}} 1$ nos da una visión inicial de los datos, mostrando el gasto en I+D como porcentaje del PIB para un grupo seleccionado de países. Por una parte, países como Finlandia y Corea presentan niveles de gasto que bordean o superan el 3\% del PIB, mientras que en otros como Argentina, Chile y Grecia dicha cifra está entre 0,4\% y 0,6\%. Así, al realizar una comparación internacional del esfuerzo dedicado a I+D nos encontramos patrones muy disímiles de gasto entre países. ¿Por qué? Claramente hay un efecto ingreso. Esto es, en la medida que una economía se hace más rica y con una mayor dotación de capital humano podrá gastar más en I+D.

$\mathrm{Al}$ respecto, Lederman y Maloney (2003) utilizan un panel de datos construidos por Lederman y Sáenz (2003) para caracterizar patrones de evolución del gasto en I+D en las diferentes etapas de desarrollo. Los autores estiman una regresión entre el gasto en $\mathrm{I}+\mathrm{D}$ como porcentaje del producto como función del logaritmo del PIB per cápita y el cuadrado de este último término. La Figura N 1 muestra los valores esperados y observados de I+D. 
CUADRO N ${ }^{\circ}$ 1: $\quad$ INVERSIÓN EN I+D EN UNA MUESTRA SELECCIONADA DE PAÍSES ${ }^{4}$

\begin{tabular}{lccr}
\hline & \multirow{2}{*}{ PIB } & \multicolumn{2}{c}{ Inversión en I+D } \\
\cline { 3 - 4 } & US millones (2005) & \% PIB & US millones \\
\hline & & & \\
Argentina & 183,309 & 0,41 & 752 \\
Chile & 115,250 & 0,61 & 703 \\
Grecia & 213,698 & 0,62 & 1.325 \\
Portugal & 173,085 & 0,78 & 1.350 \\
Brasil & 122,345 & 0,98 & 1.199 \\
España & $1.123,691$ & 1,11 & 12.473 \\
N. Zelandia & 109,041 & 1,16 & 1.265 \\
Irlanda & 196,388 & 1,18 & 2.317 \\
Rep. Checa & 122,345 & 1,27 & 1.554 \\
China & $2.228,862$ & 1,31 & 29,198 \\
Holanda & 594,756 & 1,85 & 11.003 \\
Corea & 787,625 & 2,64 & 20.793 \\
& & & \\
\hline
\end{tabular}

Fuente: Banco Mundial (2006).

Se observa una clara relación positiva entre el gasto en I+D y el log del PIB per cápita, con una particularidad: el gasto en I+D se incrementa más que proporcionalmente a medida que el PIB per cápita aumenta.

Los mismos autores encuentran que las variables que explican el mayor gasto en $\mathrm{I}+\mathrm{D}$ en los países de mayores ingresos respecto de los países pobres ${ }^{5}$ son la profundidad del sistema financiero, la protección de los derechos de propiedad intelectual, la mayor eficiencia del gobierno para movilizar recursos y la calidad de las instituciones en que se lleva a cabo investigación. Estas variables explicarían por qué los esfuerzos en I+D aumentan con el nivel de desarrollo.

La evidencia anterior permite concluir dos cosas. Primero, existe mucha divergencia en el esfuerzo en I+D realizado por los países. El grueso del gasto mundial en I+D es efectuado por economías desarrolladas. Esto refuerza la importancia de la apertura comercial, no sólo para incentivar un mayor gasto en I+D, sino también para facilitar los flujos de conocimientos generados en las economías más desarrolladas hacia el resto de los países. Segundo, el esfuerzo en I+D se correlaciona positivamente con el nivel de desarrollo de la economía.

\footnotetext{
${ }^{4}$ Dato para el último año disponible.

${ }^{5}$ A pesar de que existe evidencia de que el retorno social del gasto e I+D disminuye con el nivel de desarrollo, tal como señala en la siguiente sección.
} 
FIGURA N ${ }^{\circ}$ 1: $\quad$ GASTO I+D Y DESARROLLO

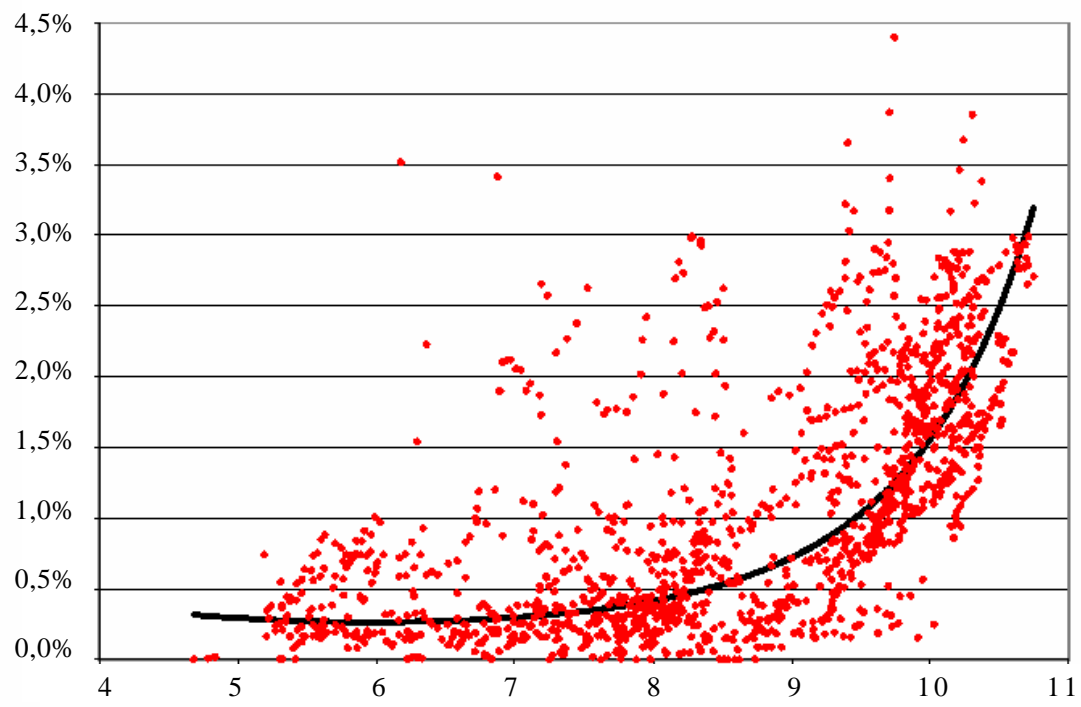

Fuente: Lederman y Maloney (2003).

Sin embargo, estas conclusiones conducen a la pregunta de si efectivamente el gasto en $\mathrm{I}+\mathrm{D}$ de los países es coherente con la rentabilidad privada y social de este tipo de actividades, o — más específicamente- si el menor esfuerzo de los países de ingresos más bajos se debe a que la inversión en I+D es menos rentable en comparación con las economías más desarrolladas. Este tema se aborda a continuación.

\subsection{Rentabilidad social y privada de I+D}

La evidencia empírica obtenida en un gran número de estudios es concluyente en señalar que la rentabilidad social de la inversión en I+D es bastante más alta que su retorno privado, la que, a su vez, es más elevada que la tasa de retorno estimada para el capital. Los estudios indican que la rentabilidad privada es muy alta, ya que esta fluctúa entre un 17\% y un 34\% (Sveikauskas, 1981 y Grilliches y Lichtenberg, 1984, respectivamente). Los retornos sociales, en tanto, serían aún más altos. La mayoría de los trabajos sobre el tema estiman tasas de rentabilidad social superior al 60\%, superando incluso el $100 \%$ en algunos casos. 
A nivel de industrias y firmas de Estados Unidos, se han reportado muy altas tasas de rentabilidad social asociadas a I+D. Grilliches y Lichtenberg (1984), por ejemplo, uno de los trabajos clásicos en este tema, estima una rentabilidad superior a $70 \%$. Otros estudios que consideran una muestra varios países, concluyen que las tasas de retorno son de ese orden o superiores ${ }^{6}$.

Por su parte, Lederman y Maloney (2003) utilizan una muestra de países durante el período 1975-2000 para calcular tasas de retornos sociales del gasto en I+D. Concluyen que la rentabilidad social del gasto en I+D sería de $78 \%$ bajo una especificación base $\mathrm{P}^{7}$. También encuentran evidencia de retornos decrecientes en I+D a medida que un país se desarrolla. Esto es, el gasto en $\mathrm{I}+\mathrm{D}$ produce incrementos de productividad mayores en países que se encuentran lejos de la frontera tecnológica respecto de los países innovadores que están moviendo la frontera. El retorno en un país promedio de la OECD estaría en un rango de $20-40 \%$. En países de ingreso medio el retorno promedio sería de alrededor de $60 \%$, mientras que en países pobres, tales como Nicaragua, el retorno promedio sería cercano a $100 \%$.

Así, los recursos dedicados a I+D son exiguos respecto del alto retorno que este tipo de inversión tiene a nivel privado y social. Esto se debe a la existencia de fallas de mercado y fallas sistémicas que inhiben el esfuerzo en I+D. Esto justifica una cierta intervención del Estado. Los tipos de instrumentos que dispone el Estado para fomentar un mayor gasto en I+D, así como los más pertinentes para el caso de Chile se abordan en la sección 4 de este trabajo.

\subsection{Financiamiento del gasto en $I+D$}

Si bien la magnitud del gasto en I+D es un indicador importante del esfuerzo innovador que realizan los países, también es importante la forma en que se financia. La evidencia internacional muestra que en la gran mayoría de los países desarrollados un porcentaje importante del gasto en I+D es financiado y ejecutado por los privados. De hecho, en los países de la OECD, las empresas privadas son responsables aproximadamente del $70 \%$ del gasto anual en $\mathrm{I}+\mathrm{D}$, mientras que las universidades, los laboratorios y centros científicos explican el 30\% restante. En dichos países, el Estado es - por lejos- el mayor financista de la investigación llevada a cabo en laboratorios y universidades, mientras que sólo contribuye con un $10 \%$ a la I+D llevada a cabo en las empresas.

\footnotetext{
${ }^{6}$ Véase, por ejemplo, Van Pottelsberghe de la Potterie y Lichtenberg (2001).

${ }^{7}$ Llegan incluso a $133 \%$ en una muestra más reducida de países.
} 
CUADRO No 2: $\quad$ FINANCIAMIENTO GASTO EN I+D EN UNA MUESTRA SELECCIONADA DE PAÍSES ${ }^{8}$

\begin{tabular}{lcccc}
\hline & Empresas & $\begin{array}{c}\text { Educación } \\
\text { superior }\end{array}$ & Gobierno & $\begin{array}{c}\text { Org. privadas sin } \\
\text { fines de lucro }\end{array}$ \\
\hline Argentina & 33 & 25 & 39,7 & \\
Chile & 35,8 & 38,8 & 11,0 & 14,3 \\
Corea & 76,7 & 9,9 & 12,1 & \\
España & 54,4 & 29,5 & 16 & \\
Finlandia & 70,1 & 19,8 & 9,5 & \\
Holanda & 57,8 & 27,9 & 14,4 & \\
Irlanda & 64,6 & 27,6 & 7,8 & \\
N. Zelandia & 42,5 & 28,5 & 28,9 & \\
Rep. Checa & 63,7 & 14,8 & 21,2 & \\
& & & & \\
\end{tabular}

Fuente: OECD y Conicyt.

FIGURA N ${ }^{\circ}$ 2: $\quad$ FINANCIAMIENTO A I+D PRIVADO EN EE.UU.

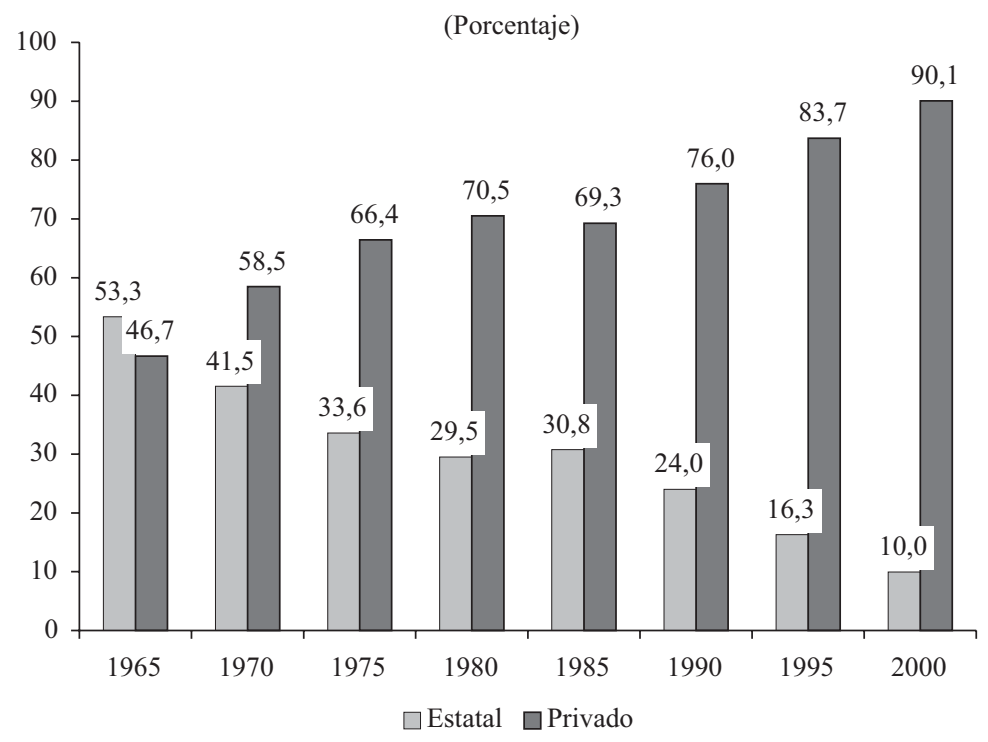

Fuente: National Science Foundation.

${ }^{8}$ Dato para el último año disponible. 
La situación contraria ocurre en países menos desarrollados. En Chile, por ejemplo, entre $25 \%$ y $36 \%$ del gasto en $\mathrm{I}+\mathrm{D}$ fue financiado por las empresas en años recientes, lo que revela que el grueso del financiamiento viene del gobierno, las universidades y las instituciones privadas sin fines de lucro (véase Cuadro $\mathrm{N}^{\circ} 2$ ). Esta situación era compartida hace no muchos años por varios de los países en que una parte muy relevante del gasto en I+D es realizado hoy por los privados. Un ejemplo notable de ello es el caso de Estados Unidos, donde se ha observado un cambio muy relevante en la composición del aporte que efectúan los distintos sectores en los últimos años, tal como se puede apreciar en la Figura $\mathrm{N}^{\circ} 2$. Similar tendencia se observa en la gran mayoría de los países innovadores, donde más del 70\% del gasto en $\mathrm{I}+\mathrm{D}$ es ejecutado hoy por la empresa privada.

\subsection{Inversión en ciencias básicas vs. investigación aplicada}

Ahora bien, si una economía decide invertir en $\mathrm{I}+\mathrm{D}$, tampoco es indiferente cómo se componga dicho gasto. Los recursos pueden destinarse a la investigación en ciencias básicas, a la investigación aplicada o al desarrollo experimental. Países exitosos en innovación, tales como Corea, Estados Unidos, Israel y Nueva Zelanda destinan 20\% o menos de los recursos dedicados a I+D a la investigación en ciencias básicas. En cambio, cerca del 60\% de la investigación en Chile se concentra en ciencias básicas o desarrollo experimental.

Así, la evidencia internacional presentada en esta sección nos revela que los países exitosos no sólo presentan un alto nivel agregado de gasto en $\mathrm{I}+\mathrm{D}$, sino también que éste es mayormente focalizado a la investigación aplicada, con una alta participación del sector privado en su financiamiento y ejecución. Esta es una buena garantía de eficiencia del esfuerzo en innovación, en términos de incrementos de productividad. En la siguiente sección se compara el esfuerzo actual de Chile en I+D con el realizado por las economías desarrolladas, se analizan las fortalezas y debilidades del Sistema Nacional de Innovación; finalmente, se recomiendan alternativas para el fomento de la I+D en Chile.

\section{Gasto de Chile en I+D}

\subsection{Diagnóstico del esfuerzo actual de Chile en I+D}

Si bien el esfuerzo de Chile en I+D en la década de los noventa más que cuadruplicó el de la década anterior, éste sigue estando muy por debajo del que realizan los países desarrollados. La brecha es aún más brutal res- 
pecto de los países innovadores, tal como quedó en evidencia en el Cuadro 1. Las cifras más recientes indican que Chile gasta alrededor de un $0,7 \%$ del PIB en I+D, del orden de US\$700 millones. Esta cifra es bastante menor comparada con los $\$ 11$ mil millones que gasta Holanda (2\% del PIB) o los casi \$18.500 millones de Corea (2,7\% del PIB).

Lederman y Maloney (2004) calculan la desviación entre el gasto efectivo en $\mathrm{I}+\mathrm{D}$ de Chile y el proyectado en base al nivel de ingreso y tamaño de la fuerza laboral del país. Los autores concluyen que Chile tiene un nivel de esfuerzo menor al que se esperaría dado su nivel de desarrollo.

Explorando otra dimensión del problema, Maloney y Rodríguez-Clare (2005) simulan el gasto en I+D en los países de la OECD si éstos presentasen un patrón de especialización similar al de Chile (principalmente en recursos naturales). Concluyen que, en promedio, los países de la OECD tendrían un $60 \%$ del gasto en I+D observado actualmente en ellos, aunque con divergencia entre los distintos países de la muestra. Por ejemplo, en países como Finlandia y Alemania, el gasto en I+D sería 30\% del gasto actual, mientras que países como Australia o Noruega dicho porcentaje sería cercano al 85\%. Dada la evidencia, los autores concluyen que el bajo esfuerzo en I+D de Chile se explica en parte por la especialización de la economía en sectores de baja intensidad en I+D. Este trabajo es sugerente respecto de la influencia de la especialización productiva en el gasto en I+D de los países. Sin embargo, no invalida las conclusiones extraídas por Lederman y Maloney (2004) respecto del bajo esfuerzo actual de Chile en materia de innovación. El patrón de especialización explica sólo parcialmente el bajo nivel de I+D en Chile. Además, en un mundo globalizado de alta competencia, los sectores de recursos naturales requieren innovar no sólo para competir mejor, sino como un imperativo de supervivencia.

En algunos sectores de la economía chilena, en donde la competencia es fuerte y existe un número significativo de jugadores, puede pensarse en fomentar las inversiones conjuntas en I+D a través de fondos públicos. Podría premiarse las actividades de innovación de consorcios de empresas - asociadas, por ejemplo a instituciones de investigación de excelencialo que apoyaría la estrategia de clusters. Este es el caso de la industria del salmón, la minería o la forestal.

De las comparaciones internacionales podemos concluir que no sólo el nivel del gasto de Chile en I+D es bajo, sino que además está distribuido inadecuadamente tanto en términos de quien lo financia como de su destino. En los países innovadores entre 60\% y 75\% del gasto en I+D es ejecutado por las empresas, lo que asegura que la investigación sea adecuada a sus necesidades $y$, por ende, que se traduzca en mayores incrementos de 
productividad. Tal como se pudo apreciar en el Cuadro $\mathrm{N}^{\circ} 2$, en Finlandia y Corea más del $70 \%$ del gasto en I+D es ejecutado por las empresas. En Chile, en cambio, esta cifra es alrededor del $36 \%$, de modo que el gobierno y las universidades realizan el grueso del esfuerzo.

Un tercer aspecto que caracteriza el esfuerzo actual en I+D de la economía chilena es la escasa focalización en ciencias aplicadas. En los países innovadores hasta el $80 \%$ del esfuerzo en I+D se focaliza en investigación aplicada, mientras que en Chile es menos de la mitad. Podría arguirse que esta estrategia es — tal vez- apropiada para un país que está cercano a la frontera del desarrollo tecnológico, pero resulta muy difícil sostenerla para uno cuyo desafío principal en materia tecnológica es adoptar y adaptar tecnologías del exterior. Lo anterior también deja en evidencia el bajo nivel de conexión entre el mundo empresarial y el académico. De hecho, tal como señalan Rodríguez y Tokman (2004), la colaboración con fines de investigación entre empresas y universidades en los países innovadores es casi un $60 \%$ más frecuente que en Chile.

En resumen, el gasto que realiza Chile en innovación es bajo, escaso en la proporción que va a investigación aplicada y con una participación insuficiente del sector privado. Además, tal como veremos en la sección 4.3., existen una serie de problemas en el Sistema Nacional de Innovación, que componen las falencias analizadas en esta sección. Lo anterior se traduce en que, en el caso de Chile, el esfuerzo actual en innovación tenga menores efectos sobre la productividad en comparación a los países líderes. De hecho, Lederman y Maloney (2003) sugieren que esta ineficiencia del Sistema Nacional de Innovación chileno se traduce en una rentabilidad social de la inversión en I+D menor respecto de países con un nivel de desarrollo similar al chileno. Una manera de cuantificar esta ineficiencia es examinando la transformación de las inversiones en I+D en patentes comerciales y comparando dicha "elasticidad" con respecto a la del promedio mundial ${ }^{9}$.

La Figura $\mathrm{N}^{\circ} 3$ muestra la elasticidad (patentes otorgadas a investigadores residentes en el país respectivo por EE.UU.)/(gasto en I+D) en comparación con el nivel promedio de los países desarrollados miembros de la Organización para la Cooperación y Desarrollo Económico (OECD). La conclusión es clara: Chile está por debajo del nivel de eficiencia promedio de los países de la OECD, aún controlando por nivel de gasto en I+D. Esta brecha sería aún más notoria si la comparación se realizara respecto de un conjunto de países innovadores.

\footnotetext{
${ }^{9}$ Bosch et al. (2003) analizan en detalle la forma de calcular estas elasticidades.
} 


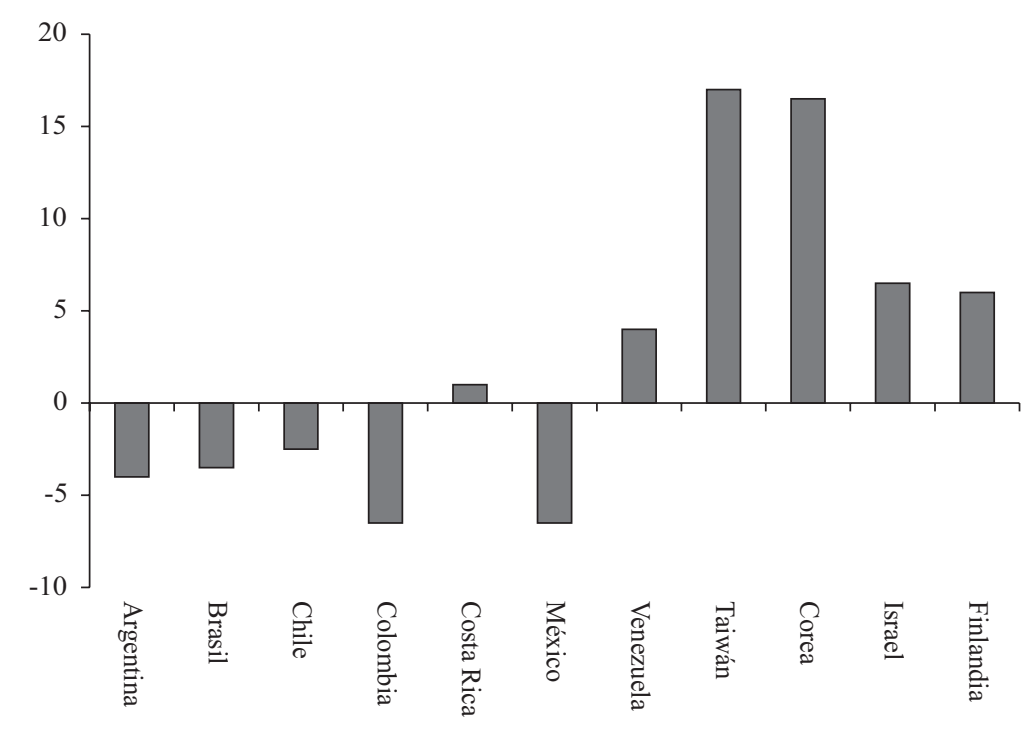

Fuente: Lederman y Maloney (2004).

\subsection{El rol del gobierno e instrumentos de apoyo a la innovación}

Antes de derivar recomendaciones de política respecto del diagnóstico realizado, se analiza brevemente la justificación económica de las políticas de promoción a la innovación por parte del Estado y los principales instrumentos de apoyo con que cuenta. En la sección siguiente se describen las principales características del sistema institucional chileno de apoyo a la innovación tecnológica.

La justificación económica para que el gobierno intervenga en el proceso de innovación mediante políticas de promoción se origina en la existencia de dos tipos de fallas. Primero, la existencia de fallas de mercado asociadas al proceso de innovación, las cuales principalmente se refieren al financiamiento del emprendimiento innovador. Segundo, la existencia de fallas sistémicas, las cuales enfatizan los problemas de coordinación entre los distintos agentes involucrados en el ámbito científico y tecnológico de un país. 


\section{Fallas de mercado}

Entre las principales fallas de mercado están, primero, la dificultad de apropiarse completamente de los beneficios derivados de la innovación. El conocimiento es un bien no rival y sólo parcialmente excluible. Esto lo convierte en un bien público, el cual es valorado positivamente por los agentes; sin embargo, los incentivos para financiarlo privadamente son limitados. Ello porque las características propias de este bien incentiva la aparición de "polizones" (free riders) que esperan aprovechar el conocimiento generado por otros sin incurrir en los costos de producción. Por ende, la inversión en conocimiento es menor a la socialmente óptima, lo cual justifica la intervención del gobierno a través de políticas de promoción. Clave en la solución de esta falla es la manera en que se definen y protegen los derechos de propiedad de las innovaciones. Segundo, debido a importantes asimetrías de información, muchas veces quienes administran los proyectos manejan un nivel de información muy superior a quienes los financian, lo cual eleva el riesgo de la inversión debido a posibles comportamientos oportunistas y, por ende, disminuyen las posibilidades de financiamiento privado.

\section{Fallas sistémicas}

Junto con las fallas de mercado, existen las denominadas fallas sistémicas, las cuales enfatizan los problemas de coordinación entre los distintos agentes involucrados en el proceso innovativo de un país. Estas fallas surgen del hecho de que el proceso de innovación incluye un conjunto de agentes y actividades que deben estar integradas para obtener un resultado exitoso. Algunas de las manifestaciones más comunes de las fallas sistémicas son: fallas en la provisión de infraestructura, fallas institucionales (por ejemplo, en lo referido a la definición y protección de los derechos de propiedad intelectual), fallas de coordinación que impiden que el sistema funcione como un todo coherente y "cuellos de botella” que se generan en el mercado de capitales o producto de una mala calidad del capital humano ${ }^{10}$.

Así, la justificación económica para la intervención del Estado en la promoción de la innovación radica en las existencias de estas fallas de mercado y sistémicas. La solución a las fallas sistémicas exige pensar sobre la institucionalidad vigente. En cuanto a las fallas de mercado, los mecanismos de solución se relacionan principalmente con el financiamiento de las actividades de innovación, además del establecimiento de normas adecuadas relacionadas con los derechos de propiedad.

\footnotetext{
${ }^{10}$ Una discusión de estos temas se encuentra en Tokman y Rodríguez (2004).
} 
Instrumentos de promoción de $I+D$

En la sección anterior se vio que existe un espacio para que el gobierno incentive un nivel de innovación más cercano al socialmente óptimo. Los principales instrumentos para promover un mayor esfuerzo en I+D son:

- Instrumentos directos, tales como subsidios a las empresas que realizan I+D, subvenciones, préstamos, co-financiamiento a proyectos y financiamiento de laboratorios públicos de I+D y de investigación universitaria.

- Instrumentos indirectos, tal como incentivos tributarios. Podemos distinguir tres tipos de incentivos tributarios aplicables para fomentar el gasto en I+D:

- Deducciones de gasto corriente, que incluye la amortización de gastos corrientes en $\mathrm{I}+\mathrm{D}$, los cuales pueden ser contablemente cargados a gastos y deducidos del ingreso tributable en el año corriente.

- Depreciación acelerada de las inversiones en máquinas, equipos y edificios para actividades de $\mathrm{I}+\mathrm{D}$

- Programas de exención tributaria, que autorizan a las firmas a decidir libremente acerca de los proyectos de I+D que desean financiar. La exención tributaria sobre el gasto en $\mathrm{I}+\mathrm{D}$ puede aplicarse de dos maneras diferentes: un esquema de volumen o un esquema incremental. Bajo el primer esquema los descuentos tributarios compensan el monto completo de los gastos acarreados en actividades de I+D. Bajo el segundo esquema, los descuentos tributarios compensan sólo a la cantidad adicional de gastos elegibles de I+D. La base de referencia para el incremento es generalmente la diferencia nominal respecto al promedio de gasto en I+D de los dos o tres últimos años (incremental rotativo). Otras prácticas menos usuales toman como base un monto fijo (incremental fijo) o la relación entre I+D y ventas (incremental indexado a ventas) donde la empresa puede descontar de impuestos los gastos de I+D cuando éstos constituyen un mayor porcentaje de las ventas que en el año en que se fijó la base. Entre las ventajas de un esquema de volumen se encuentra la mayor simpleza de elaborar, administrar y de aplicar y el hecho de que son más fáciles de entender y calcular por las empresas. Sin embargo, son más costosos para el Estado y podrían subsidiar I+D que las empresas habrían realizado de todas maneras. 
Por su parte, los esquemas incrementales motivan a invertir por sobre lo histórico y tienen un menor impacto económico sobre el Estado. Las desventaja de estos tipos de incentivos es que son más complejos y difíciles de usar, puede tener pocos beneficios sobre las empresas con presupuestos estables de I+D (por ejemplo, en firmas grandes).

La evidencia internacional muestra que, con excepción de algunos países de Europa Oriental, el apoyo público directo a la I+D de las empresas ha ido en descenso en los países de la OECD, fomentándose más las medidas indirectas, tales como los incentivos tributarios. Entre 2002 y 2004, Bélgica, Irlanda y Noruega establecieron un nuevo esquema de incentivos tributarios, lo que lleva a 18 el número de países de la OECD que emplean este sistema para potenciar la I+D. El Reino Unido también instauró incentivos tributarios para las grandes empresas y completó su esquema para las pequeñas.

En la mayoría de los países de la OECD, la base para el incentivo tributario son los gastos corrientes en I+D. Algunos países incorporan también las máquinas, equipos y edificios. Casos particulares son Holanda y Bélgica donde los incentivos fiscales se aplican sólo a los salarios del personal calificado de I+D. Por su parte, 9 de los países de la OECD ofrecen depreciación acelerada especial de maquinaria y equipos utilizados en actividades de $\mathrm{I}+\mathrm{D}$

Adicionalmente, los países se están esforzando por estimular el espíritu empresarial e impulsar las actividades de I+D en las pequeñas y medianas empresas (Pymes), mediante, por ejemplo, el apoyo al capital de riesgo, la asistencia preferente a las Pymes y otros incentivos especiales.

Por último, cinco países (Canadá, Noruega, Japón, España y el Reino Unido) ofrecen estímulos a la I+D de proyectos conjuntos entre empresas y universidades o entre empresas y centros de investigación.

Así, la teoría y evidencia respecto a la conveniencia de los incentivos tributarios no es concluyente. La principal ventaja de los incentivos tributarios respecto de mecanismos más directo, tales como son los subsidios, se refiere a su neutralidad. Esto es, las empresas son quienes deciden cómo, cuándo y cuánto invertir, en vez de que esto sea determinado a través de una autoridad central. Con ello, se permite que sea el propio mercado el que decida dónde se encuentran las mayores oportunidades de mejorar la competitividad. Asimismo, los costos administrativos y burocráticos asociados a un sistema de incentivos tributarios podrían ser menores a los de un régimen de subsidios. 
Por su parte, la utilización de subsidios tendría la ventaja de mantener la simplicidad del sistema tributario. Además, le permitiría al gobierno incentivar proyectos donde se espera que haya mayores efectos de spillover y, por ende, permitiría una utilización más efectiva y focalizada de los recursos públicos.

Parece no existir una receta óptima para la promoción de las actividades de innovación. La evaluación sobre la conveniencia de aplicar uno u otro tipo de incentivos debe considerar qué tipo de fallas de mercado o sistémicas adquieren mayor protagonismo en un determinado país. Por ende, antes de establecer recomendaciones para el fomento de la I+D en Chile, describiremos el sistema institucional chileno de apoyo a la innovación tecnológica, lo cual nos dará algunas luces respecto de las principales fallas de mercado y sistémicas presentes. Ello, a su vez, nos guiará en las propuestas para fomentar la I+D en Chile.

\subsection{Sistema institucional chileno para el apoyo a la innovación tecnológica}

El Sistema Nacional de Innovación está integrado por las empresas, universidades, centros de investigación, agencias públicas, y en general todas las organizaciones privadas o públicas que contribuyen mediante la creación, adaptación y adopción de tecnologías a incrementar el nivel global de conocimiento del país. En el caso chileno, este sistema, si bien no está diseñado ni constituido formalmente como tal, sí opera en la práctica.

Entre los agentes públicos más relevantes en el diseño de políticas se encuentran el Ministerio de Agricultura, Ministerio de Educación, el Ministerio de Economía y MIDEPLAN, este último a cargo de la Iniciativa Científica Milenio, que financia institutos científicos y otorga becas de investigación. De acuerdo al presupuesto nacional para el 2004 destacan dos grandes fuentes de financiamiento a actividades de I+D e innovación tecnológica. La primera consiste en recursos públicos destinados a financiar directamente la oferta, es decir, universidades e institutos tecnológicos públicos. Entre estos tenemos: el Instituto de Investigaciones Agropecuarias (INIA), el Instituto Forestal de Chile (INFOR), el Centro de Información de Recursos Naturales (CIREN), el INN, el Instituto de Fomento Pesquero (IFOP), la Comisión Chilena de Energía Nuclear (CCHEN), el Servicio Hidrográfico y Oceanográfico de la Armada, el Instituto Geográfico Militar y el Instituto Antártico Chileno (INACH). La segunda consiste en la canalización de recursos a través de los fondos tecnológicos, cuya operación depende de diferentes ministerios. Entre los principales fondos tenemos: el Fondo Nacional de Desarrollo Científico y Tecnológico o FONDECYT (bajo 
CUADRO N 3: $\quad$ RECURSOS PÚBLICOS PARA CIENCIA Y TECNOLOGÍA (2004)

(Mill. \$)

\section{AGRICULTURA}

Fundación para la Innovación Agraria (FIA) 3.466

$\begin{array}{ll}\text { INIA } & 7.191\end{array}$

INFOR (Subsecretaría de Agricultura) $\quad 924$

CIREN (Subsecretaría de Agricultura) 418

$\begin{array}{ll}\text { Fundación Chile } & 895\end{array}$

\section{ECONOMÍA}

FONTEC

Fondo de Desarrollo e Innovación (FDI, CORFO)

Fondo Innovación Tecnológica Bío-Bío 504

Programa de Desarrollo e Innovación Tecnológica

Programa de Desarrollo e Innovación Tecnológica 1.664

$\begin{array}{lr}\text { Subsecretaría de Agricultura (FIA) } & 569\end{array}$

$\begin{array}{ll}\text { CONICYT } & 1.408\end{array}$

Fundación Chile $\quad 330$

INN $\quad 393$

$\begin{array}{ll}\text { Programa de Marcas y Patentes } & 261\end{array}$

Fondo de Investigación Pesquera (Subsecretaría de Pesca) 2.211

$\begin{array}{ll}\text { IFOP (Subsecretaría de Pesca) } & 392\end{array}$

$\begin{array}{ll}\text { Fundación Chile (CORFO) } & 713\end{array}$

$\begin{array}{ll}\text { Profo y Fat } & 13.484\end{array}$

\section{EDUCACION}

FONDECYT (CONICYT) 21.263

$\begin{array}{lr}\text { FONDEF (CONICYT) } & 9.900\end{array}$

Becas Nacionales de Posgrado (CONICYT) 3.059

Programa de Ciencias para la Economía del Conocimiento (Banco Mundial) 5.129

$\begin{array}{ll}\text { Programa Explora (CONICYT) } & 723\end{array}$

Instituto Astronómico Isaac Newton $\quad 55$

Fondo de Desarrollo Institucional $\quad 8.313$

$\begin{array}{lr}\text { Fondo de Desarrollo Institucional-Infraestructura } & 16.375\end{array}$

MIDEPLAN

$\begin{array}{lr}\text { Programa Iniciativa Científica Millenium } & 3.610\end{array}$

$\begin{array}{ll}\text { Programa de Becas } & 5.180\end{array}$

\section{MINERÍA}

Comisión Chilena de Energía Nuclear $\quad 3.979$

SERNEAGEOMIN $\quad 4.060$

DEFENSA

Servicio Hidrográfico u Oceanográfico de la Armada de Chile 2.563

$\begin{array}{lr}\text { Instituto Geográfico Militar } & 1.102\end{array}$

$\begin{array}{ll}\text { Servicio Aerofotogramétrico de la FACH } & 407\end{array}$

\section{RELACIONES EXTERIORES}

Instituto Antártico Chileno $\quad 1.926$

TOTAL (Mill. \$) $\quad 138.438$

TOTAL (Mill. US\$) 222

Fuente: Benavente (2004), en base a información de DIPRES. 
CONICYT, en el Ministerio de Educación), el cual financia investigación básica no necesariamente aplicada ni comercializable; el programa Innova de CORFO, destinado al financiamiento de actividades de innovación en la empresa, el cual financia proyectos innovadores con elevadas externalidades; y el FONDEF (bajo CONICYT, en el Ministerio de Educación) que financia proyectos asociativos en torno a innovaciones tecnológicas. Adicionalmente, los recursos públicos incluyen becas de posgrado tanto en Chile como en el exterior. El siguiente cuadro presenta las distintas fuentes de recursos públicos según ministerio para ciencia y tecnología.

Por último, es necesario precisar que el sistema tributario chileno no provee incentivos especiales a la $\mathrm{I}+\mathrm{D}$. Los gastos de $\mathrm{I}+\mathrm{D}$ tienen el mismo tratamiento que otros gastos de la empresa (Artículo 33, Ley de Impuesto a la Renta).

\subsection{Propuestas para fomentar la I+D en Chile}

Para promover la I+D en Chile es probablemente adecuado pensar en una combinación entre políticas de incentivos tributarios y financiamiento directo. Hoy en día, tal como vimos en la sección anterior, el sistema tributario chileno no provee de reglas especiales a la I+D. En cambio, la mayoría de las políticas de fomento a la I+D se realizan a través de los fondos concursables. Por ende, las actuales fuentes de financiamiento directo deben ser complementadas con políticas de incentivos tributarios a la I+D de las empresas.

\section{Criterios de diseño de incentivos tributarios}

Para que los incentivos tributarios a la $\mathrm{I}+\mathrm{D}$ de las empresas logren su objetivo se deben cumplir los siguientes criterios de diseño:

Primero, para que el incentivo tributario sea efectivo debe ser simple, consistente, confiable, predecible y presentar bajos costos administrativos. Esto es, sistemas demasiados complejos o muy cambiantes desincentivan las inversiones en I+D pues las empresas requieren certidumbre para planificar sus gastos en I+D. Además, para que el incentivo sea efectivo, los costos administrativos se deben mantener lo más bajos posibles, en especial en el caso de las pequeñas y medianas empresas (Pymes).

Segundo, se debe definir claramente el concepto de $\mathrm{I}+\mathrm{D}$ y gastos que dan derecho al incentivo. Los gastos que dan derecho al incentivo tributario por I+D deben reflejar bien el patrón de gastos generalmente incu- 
rridos al realizar I+D. Tercero, se deben simplificar al máximo las posibles excepciones y casos particulares. Por último, los incentivos tributarios no deben traducirse en una carga presupuestaria excesiva para el Estado.

Incentivos a la $I+D$

Una propuesta de incentivo a la I+D en Chile consiste en complementar los actuales fondos concursables con un crédito tributario para la actividad de I+D. Esto es, se propone implementar un sistema que permita a las empresas rebajar directamente de impuestos — digamos— el $40 \%$ de los recursos gastados en I+D contra el impuesto de Primera Categoría (descuento tributario en volumen). La deducción sería del Impuesto de Primera Categoría que deben pagar en el mismo ejercicio en que se incurre en los gastos del contrato de $\mathrm{I}+\mathrm{D}$, siendo el remanente aprovechado como crédito contra el Impuesto de Primera Categoría de los ejercicios siguientes, existiendo la posibilidad de diferir el beneficio indefinidamente.

Por su parte, el Ministerio de Economía debiera confeccionar una lista de las organizaciones sin fines de lucro con las cuales se puede contratar la I+D, siendo también la entidad encargada de auditar los resultados. Integrarían esta lista de pleno derecho las universidades reconocidas por el Estado, que acrediten al Ministerio estar capacitadas para el desarrollo de actividades de I+D. El Servicio de Impuestos Internos, a través de sus procesos normales de fiscalización, sería el responsable de fiscalizar la información entregada por las empresas, que contendría la declaración de los gastos en I+D, una vez que ya han sido incurridos.

Adicionalmente, y como complemento a esta propuesta, podría destinarse parte de los recursos dedicados a este fin para premiar las innovaciones que efectivamente se materializan. Para contabilizar el éxito de la innovación, pueden utilizarse las patentes generadas (en caso de que la innovación sea patentable).

Tal como se dijo previamente, es importante definir claramente el concepto de I+D y gastos que dan derecho al incentivo. Para ello, se propone utilizar la definición dada por el Manual Frascati (OECD), el cual clasifica la I+D en tres tipos de actividad: investigación básica, investigación aplicada y desarrollo. Los gastos en I+D se definirían como gastos corrientes, por ejemplo, en sueldos, asesorías, materiales e insumos, activos fijos (excepto bienes raíces) y gastos de compra de licencias y registro de patentes comerciales. 


\section{Efectos esperados}

La implementación de un incentivo tributario de este tipo estimularía un esfuerzo adicional en I+D a través de un incremento en el gasto en dichas actividades ejecutado y financiado por las propias empresas. Además, permitiría complementar los actuales incentivos estatales directos a la $\mathrm{I}+\mathrm{D}$ con incentivos indirectos menos discrecionales que permitan al propio mercado decidir dónde se encuentran las mayores oportunidades de mejorar la competitividad. Por su parte, permitiría también una mayor orientación de la I+D hacia el mercado y fomentaría la investigación aplicada. En definitiva, todo esto repercutiría en un mayor desarrollo científico-tecnológico en el sector privado, que impactará positivamente la productividad, competitividad y, por ende, el crecimiento económico del país. Esto es particularmente importante en el contexto de un mundo globalizado y competitivo en el cual la economía chilena está cada vez más inserta.

¿De dónde se obtendrían los recursos fiscales necesarios para financiar esta medida? El royalty a la minería generará recursos por algunos cientos de millones para actividades afines a I+D. Una alternativa es canalizar parte de los recursos del royalty a la minería a través del crédito tributario propuesto para la actividad de I+D, siendo el porcentaje restante canalizado a través de concursos públicos.

Adicionalmente, el mayor esfuerzo de I+D ejecutado por el sector privado debiera tener un impacto positivo sobre la productividad de las empresas que realizan directamente el gasto en I+D y de terceras empresas que se benefician indirectamente a través del derrame de conocimientos hacia ellas. Esto, a su vez, impactaría positivamente las utilidades y los flujos sujetos a impuesto de estas empresas. Por ende, desde el punto de vista fiscal esta medida no debiera traducirse en una carga adicional para el fisco. La recaudación adicional de impuestos derivados del aumento de las utilidades de las empresas, junto con la utilización de recursos del royalty a la minería, debieran más que compensar la disminución inicial en impuestos.

\section{Propuestas adicionales para mejorar el SNI chileno}

Si bien en Chile existen fallas de mercado que requieren ser solucionadas mediante un mayor financiamiento proveniente de fondos concursables o incentivos indirectos tales como el propuesto anteriormente, se visualizan también importantes fallas sistémicas. Específicamente, se observa 
la ausencia de una política científico-tecnológica a nivel nacional, lo cual finalmente se traduce en un Sistema Nacional de Innovación fragmentado y que evidencia fallas de coordinación, con muchos actores y programas que operan de manera aislada. Esto genera amplios espacios para la duplicación de esfuerzos y desaprovechamiento de economías de escala y de ámbito.

Por ende, cualquier sugerencia de política debiera incluir un plan nacional que otorgue una articulación y coordinación de las instituciones que componen el Sistema Nacional de Innovación (SNI) chileno y que priorice objetivos para mejorar la eficiencia del esfuerzo nacional en I+D. Para ello, la creación de una institución líder es esencial para eliminar esta falla sistémica presente en nuestra economía en materia de innovación. Esta institución debiera coordinar los esfuerzos que cada agente involucrado realiza en materia de innovación y definir claramente las políticas necesarias para incrementar la eficiencia del SNI chileno. El Consejo Nacional de Innovación para la Competitividad, creado a fines de 2005, puede avanzar en esta dirección, pero aún es muy temprano para evaluar su accionar.

$\mathrm{Al}$ respecto, en los países exitosos en innovación existen consejos o ministerios creados especialmente para este fin. Por ejemplo, Israel desde 1969 orienta su política tecnológica a través de la oficina del Chief Scientist, mientras que Corea lo hace a través del Ministry of Science and Technology. Otro modelo interesante es el Science and Technology Policy Council de Finlandia. En Chile esta institucionalidad no existe y la consecuencia de esto son traslapes y falta de coordinación entre los distintos agentes, fondos y políticas relacionados con la innovación.

Adicionalmente, es necesario acrecentar los lazos entre las universidades que hacen investigación y las empresas. Hoy en día, las universidades no generan necesariamente conocimiento innovativo y muchas empresas carecen de estructuras que permitan que el conocimiento generado en la universidad sea puesto en la práctica. Existe evidencia que muestra que los contratos de cooperación entre las universidades y las empresas son socialmente beneficiosos, aunque la existencia de externalidades impide que dichos contratos surjan de mutuo acuerdo. Por ende, el Estado tiene un rol que cumplir en la promoción de su existencia. Una opción es promover la existencia de instituciones intermediadoras en el mercado tecnológico, tal como proponen Bitrán (2004) y Benavente (2004). Dichos organismos debieran orientarse en la promoción de las aplicaciones comerciales de los resultados de la investigación universitaria. En Chile algunas universidades, como la Pontificia Universidad Católica de Chile (a través de su programa UC- Empresa) han tomado en serio el desafío de acercarse al mundo empre- 
sarial y fomentar el emprendimiento. Pero el esfuerzo global es aún muy insuficiente.

También es indispensable para la eficiencia del sistema de innovación chileno mantener una clara definición y protección de los derechos de propiedad intelectual y el fomento de la apertura comercial y la competencia interna de manera de incentivar el uso de procesos de frontera en las empresas. Junto con ello, la mayor calificación del capital humano es clave para incentivar la innovación y difusión tecnológica. Sin una oferta de capital humano calificado, la capacidad innovadora de la economía se ve limitada al convertirse el trabajo en un débil complemento del capital.

Junto con las políticas anteriores, resulta importante contar con un mercado laboral flexible para potenciar la innovación. Una propuesta para lograr este objetivo es facilitar el teletrabajo o trabajo a distancia, que se lleva a cabo desde el hogar a través de medios informáticos. Hoy existe una dotación significativa de capital humano que — debido a la presencia de restricciones - no se utiliza en la producción ni la innovación. Una legislación laboral flexible al respecto promovería la innovación en los procesos productivos de las empresas y tendría, adicionalmente, otros efectos positivos sobre la economía: permitiría a ciertos grupos (como las madres y dueñas de casa) incorporarse más plenamente al trabajo y aumentar los ingresos familiares.

Cabe mencionar que algunas de estas propuestas ya han sido consideradas por el actual Gobierno de Chile. El Plan Chile Compite, anunciado por el ministro de Hacienda a fines de julio de 2006, incorpora incentivos orientados a fomentar la innovación y el desarrollo de tecnologías. Para lograr esto el Plan propone las siguientes medidas:

- Incentivo a la adopción de nuevas tecnologías. Esta medida considera uniformar en un 15\% la tasa de Impuesto Adicional que grava los pagos al extranjero por concepto de fórmulas, patentes, trabajos de ingeniería y asesorías técnicas. Con ésta el gravamen a la importación de software quedará en 15\%.

- Crédito tributario a inversión privada en I+D. La medida considera la creación de un crédito tributario de 35\% sobre la inversión en I+D por parte de las empresas privadas que se haga en conjunto con universidades y centros de investigación acreditados. Además, la propuesta considera la incorporación del monto no subsidiado como gasto de la empresa para fines tributarios. 


\section{Conclusiones}

La literatura empírica de crecimiento es enfática en señalar la importancia del gasto en I+D sobre el crecimiento económico de un país. Un mayor esfuerzo en I+D contribuye a incrementar los niveles de productividad de una economía y, con ello, fomenta el crecimiento de los países.

Mayor controversia existe respecto del tipo de instrumento estatal más adecuado para fomentar mayores niveles de innovación. Esto pues la política más eficiente depende de características propias de la economía en cuestión. En Chile, la mayoría de las políticas de incentivos la constituyen los fondos concursables. Por el contrario, no existen incentivos tributarios para I+D. En este trabajo se argumenta a favor de complementar los actuales incentivos directos a la I+D con créditos tributarios a los gastos en I+D. La ventaja de este tipo de incentivos radica en su neutralidad. Esto es, las empresas son quienes deciden cómo, cuándo y cuánto invertir, en vez de que esto sea determinado a través de una autoridad central. Con ello, se permite que sea el propio mercado el que decida dónde se encuentran las mayores oportunidades de mejorar la competitividad. Asimismo, presentan una buena relación costo-beneficio para el Estado.

Adicionalmente, para que el mayor esfuerzo en I+D se traduzca en incrementos de productividad es clave un funcionamiento coordinado y articulado del SNI. Para ello, resulta fundamental la creación de una institución líder que coordine los esfuerzos que cada agente involucrado realiza en materia de innovación y que defina claramente las políticas necesarias para incrementar los niveles de innovación de la economía. Esto es esencial para eliminar las actuales fallas sistémicas que presenta el SNI chileno.

Por último, es indispensable para la eficiencia del SNI chileno mantener una clara definición y protección de los derechos de propiedad intelectual y el fomento de la apertura comercial y la competencia interna de manera de incentivar en las empresas el uso de procesos de frontera. Junto con ello, la mayor calificación del capital humano es clave para la adopción de nuevas tecnologías.

Un proyecto reciente del Gobierno presentado en julio de 2006, el Plan Chile Compite, avanza en algunos de estos frentes, al establecer un crédito tributario de 35\% a la inversión en I+D realizada por empresas privadas en conjunto con universidades y otros centros de investigación acreditados. 


\section{REFERENCIAS}

Aghion, P., Harris, C., Howitt, P. y J. Vickers (2001): “Competition, Imitation and Growth with Step-by-Step Innovation”. En Review of Economics Studies, 68, pp. 467-492.

Aghion, P. y P. Howitt (1992): “A Model of Growth through Creative Destruction”. En Econométrica, 60, pp. 323-351.

Banco Mundial (2006): World Development Indicators.

Bayoumi, T., D. Coe y E. Helpman (1999): “R\&D Spillovers and Global Growth”. En Journal of International Economics, 47, pp. 399-428.

Benavente, J. M. (2004): “Innovación Tecnológica en Chile. Dónde Estamos y Qué Se Puede Hacer”. Documento de Trabajo N ${ }^{\circ} 295$, Banco Central de Chile.

Benavente, J. M. (2004): “Cooperación Tecnológica entre Universidades y Empresas: Qué Son, Cómo Operan y Cuál Es su Impacto en Chile”. En Foco 21, Expansiva, Santiago.

Bergoeing, R. y A. Repetto (2004): “Copiar No Es Malo: Competencia, Adopción e Innovación”. En Foco 19, Expansiva, Santiago.

Bitrán, E. (2004): "Sistema de Innovación, Consorcios Tecnológicos y Clusters Dinámicos en Chile”. En Foco 20, Expansiva, Santiago.

Bosch, M., Lederman, D. y W. Maloney (2003): "Patenting and Efficiency: A Global View”. Documento mimeografiado. Office of the Chief Economist for LCR, Banco Mundial, Washington, DC.

Cameron, G., J. Proudman y S. Redding (2005): “Technological Convergence, R\&D, Trade and Productivity Growth”. En European Economic Review, 49, pp. 775-807.

Coe, D. y E. Helpman (1995): “International R\&D Spillovers”. En European Economic Review 39, pp. 859-887.

Coe, D., Helpman, E. y Hoffmaister, W. (1997): "North-South R\&D Spillovers". En Economic Journal 107, pp. 134-149.

Dollar, D. y E. Wolf (1997): "Convergence of Industry Labor Productivity among Advanced Economies, 1963-1982”. En Edward N. Wolf (ed.), The Economics of Productivity. United Kingdom: Elgar.

Easterly, W. y R. Levine (2002): "It is Not Factor Accumulation: Stylized Facts and Growth Models”. En N. Loayza y R. Soto (eds.), Economic Growth: Sources, Trends and Cycles. Banco Central de Chile.

Griffith, R., S. Redding y J. Van Reenen (2004): "Mapping the Two Faces of R\&D: Productivity Growth in a Panel of OECD Industries". En Review of Economics and Statistics, 86(4), pp. 883-895.

Grilliches, Z. y F. Lichtenberg (1984): "Interindustry Technology Flows and Productivity Growth: A Reexamination”. En Review of Economics and Statistics, 66 (2), 324329.

Grossman, G. y E. Helpman (1991a): “Quality Ladders in the Theory of Growth”. En Review of Economics Studies, 58, pp. 43-61.

Grossman, G. y E. Helpman (1991b): "Innovation and Growth in the Global Economy”. Cambridge: MIT Press.

Hall, R. y C. Jones (1999): "Why do Some Countries Produce So Much More Output Per Worker Than Others?” En Quaterly Journal of Economics, 114 (1), pp. 83-116.

Hall, R. y van Reenen (2000): "How Effective Are Fiscal Incentives for R\&D? A Review of the Evidence”. En Research Policy, 29 (4), pp. 449-469. 
Larraín, F. (2006): “Innovación en Chile: Análisis y Propuestas”. Serie de Estudios Económicos y Sociales, Banco Interamericano de Desarrollo, julio.

Lichtenberg, F. y D. Siegel (1991): “The Impact of R\&D Investment on Productivity New Evidence Using Linked R\&D-LRD Data”. En Economic Inquirity, 29 (2), pp. 203-229.

Keller, W. (2001): “Knowledge Spillovers at the World’s Technology Frontier”. Discussion Paper $\mathrm{N}^{\circ}$ 2815, CEPR.

Larraín, F. (2006): “Innovación en Chile: Análisis y Propuestas”. Documento RE1-06-002, Serie de Estudios Económicos y Sociales, Banco Interamericano de Desarrollo, julio.

Lederman, D. y W. Maloney (2003): "R\&D and Development”. World Bank Research Working Paper 3024.

Lederman, D. y W. Maloney (2004): “Innovación en Chile: ¿Dónde Estamos?”. En Foco 18, Expansiva, Santiago.

Lederman, D. y L. Sáenz (2003): “Innovation around the World: A Cross-Country Data Base of Innovation Indicators”. Mimeo. Office of the Chief Economist for LCR, Banco Mundial, Washington, D.C.

Maloney, W. y A. Rodríguez-Clare (2005): "Innovation Shortfalls”. Washington, D.C., Banco Mundial, Washington D.C.

Rodríguez J. y M. Tokman (2004): "Economía del Conocimiento para un Crecimiento Sostenido”. En Foco 46, Expansiva, Santiago.

Romer, P. (1986): "Increasing Returns and Long-Run Growth”. En Journal of Political Economy, 94, pp. 1002-1037.

Romer, P. (1990): “Endogenous Technological Change”. En Journal of Political Economy, 98: S71-S102.

Sveikauskas, L. (1981): “Technological Inputs and Multifactor Productivity Growth”. En Review of Economics and Statistics, LXII, 275-282.

Van Pottelsberghe de la Potterie y F. Lichtenberg (2001): “Does Foreign Direct Investment Transfer Technology across Borders?” En Review of Economics and Statistics, 83, pp. 490-97. 